ARTICLE

\title{
Ferroelectric columnar assemblies from the bowl- to-bowl inversion of aromatic cores
}

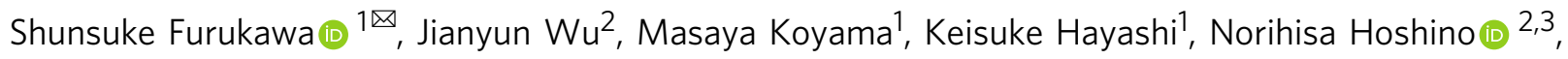

Takashi Takeda (1) ${ }^{2,3}$, Yasutaka Suzuki ${ }^{4}$, Jun Kawamata ${ }^{4}$, Masaichi Saito (i) ${ }^{1 凶}$ \& Tomoyuki Akutagawa (i) ${ }^{2,3 凶}$

Organic ferroelectrics, in which the constituent molecules retain remanent polarization, represent an important topic in condensed-matter science, and their attractive properties, which include lightness, flexibility, and non-toxicity, are of potential use in state-of-the-art ferroelectric devices. However, the mechanisms for the generation of ferroelectricity in such organic compounds remain limited to a few representative concepts, which has hitherto severely hampered progress in this area. Here, we demonstrate that a bowl-to-bowl inversion of a relatively small organic molecule with a bowl-shaped $\pi$-aromatic core generates ferroelectric dipole relaxation. The present results thus reveal an unprecedented concept to produce ferroelectricity in small organic molecules, which can be expected to strongly impact materials science.

\footnotetext{
${ }^{1}$ Department of Chemistry, Graduate School of Science and Engineering, Saitama University, Shimo-okubo, Sakura-ku, Saitama-city, Saitama 338-8570, Japan. ${ }^{2}$ Graduate School of Engineering, Tohoku University, Sendai 980-8579, Japan. ${ }^{3}$ Institute of Multidisciplinary Research for Advanced Materials (IMRAM), Tohoku University, 2-1-1 Katahira, Aoba-ku, Sendai 980-8577, Japan. ${ }^{4}$ Graduate School of Sciences and Technology for Innovation, Yamaguchi University, 1677-1 Yoshida, Yamaguchi 753-8512, Japan. ${ }^{凶}$ email: furukawa@mail.saitama-u.ac.jp; masaichi@chem.saitama-u.ac.jp; akutagawa@tohoku.ac.jp
} 
F erroelectric materials, which undergo reversible electric polarization upon exposure to an external electric field, are crucial components of ferroelectric devices, such as nonvolatile memories, actuators, and piezoelectric devices ${ }^{1}$. The ferroelectricity of organic molecules has attracted attention as a promising alternative to inorganic ferroelectric materials that contain precious and/or toxic metals $s^{2,3}$. However, examples of organic ferroelectrics are relatively scarce, and those known typically present one of the following three mechanisms: (1) orientational change of polar low-molecular-weight compounds ${ }^{4-6}$, (2) electron transfer in donor-acceptor-type chargetransfer complexes ${ }^{7-9}$, and (3) proton transfer in hydrogenbonding networks ${ }^{10-12}$ (Fig. 1, left). Therefore, the development of new strategies for designing organic ferroelectrics is of fundamental importance and represents a primordial step for versatile memory applications. Chemical design strategies based on $\pi$-electron systems afford control over electrical conduction, as well as magnetic and optical properties. For instance, the chargeordered state of the cation radical salts of bis(ethylenedithio)tetrathiafulvalene leads to electronic polarization-type ferreoelectrics $^{13}$. On the contrary, both the flexibility and the dynamics of molecular assemblies of low-molecular-weight compounds offer an interesting dynamic environment that may allow controlling certain physical properties, such as dislocation, ferroelectricity, and ferroelasticity. Indeed, ferroelectric responses have been observed in the flip-flop motion of a supramolecular cation and the multi-axial rotation of polar molecules in a plastic crystalline state ${ }^{6,14}$. Such diverse motional freedom in molecular assemblies could potentially be exploited to fabricate functional ferroelectric molecular memory materials. The supramolecular assembly of liquid crystalline compounds in a bowl-shaped structure induces dipole moments that subsequently lead to ferroelectricity (Fig. 1, middle) ${ }^{15,16}$. Although bowl inversions have typically been observed in solution, ferroelectricity that originates from bowl inversion in supramolecular assemblies has not yet been realized, even though some advances using bowl-shaped molecules have been reported ${ }^{17-19}$. For example, subphthalocyanine derivatives with strong axial dipoles have shown permanent homeotropic alignment and polarization in columnar liquid crystals with electric fields ${ }^{20}$ and ferroelectric properties in nematic phases ${ }^{21}$. On the other hand, the use of polar oligo-(vinylidenedifluoride) and/or amide side chains are also useful to induce ferroelectricity in solid-state columnar organic $\pi$-conjugated molecules ${ }^{22,23}$. A bowl-to-bowl inversion mechanism for the ferroelectric response is hardly designed in solid-state columnar assembly, necessitating the utilization of highly thermal fluctuated liquid crystal or plastic crystal states.

Here, we report organic ferroelectric materials based on hexaalkoxy trithiasumanenes (CnSS), which exhibit invertible bowl-shaped $\pi$-aromatic cores. We discovered that the bowlshaped aromatic cores of these CnSS molecules are onedimensionally stacked in a columnar fashion, and that the dipole moment originating from the aligned bowl-shaped framework leads to remanent polarization in the crystals. The present work thus describes a concept of invertible aromatic cores for materials design that provides facile access to solutionprocessable and low-cost single-columnar ferroelectric memory devices with a high density $\left(>12 \mathrm{~TB} \mathrm{~cm}^{-2}\right)$.

\section{Results}

Molecular design and synthesis. We selected trithiasumanene $(\mathbf{S S})^{24}$ as the bowl-shaped $\pi$-aromatic core, given its low bowlinversion barrier. In general, the magnitude of the bowl-inversion barrier $\left(\Delta E_{\mathrm{b}}\right)$ is closely related to the bowl depth $\left(l_{\mathrm{b}}\right)$ of the curved $\pi$-frameworks. For the typical bowl-shaped molecule sumanene $\left(l_{\mathrm{b}}=1.143 \AA ; \Delta E_{\mathrm{b}}=18.2 \mathrm{kcal} \mathrm{mol}^{-1}\right)^{25}$, these parameters are higher than those of corannulene $\left(l_{\mathrm{b}}=0.877 \AA\right.$ and $\Delta E_{\mathrm{b}}=9.2$ $\left.\mathrm{kcal} \mathrm{mol}^{-1}\right)^{26}$ (Fig. 2). The introduction of large heteroatoms, such as sulfur and selenium in curved $\pi$-molecules affords heterasumanenes, whereby the dynamic and electronic properties can be tuned by judicious choice of the inserted heteroatom(s $)^{27-31}$. Among these, SS molecules that bear three $\mathrm{S}$ atoms in the sumanene $\pi$-skeleton exhibit even smaller values $\left(l_{\mathrm{b}}=0.76 \AA\right.$ and $\left.\Delta E_{\mathrm{b}}=1.9 \mathrm{kcal} \mathrm{mol}^{-1}\right)$ than those of sumanene and corannulene $e^{25}$. The large atomic radius of the S-atom leads to a reduction of both parameters for the $\pi$-framework, whereas Seatoms, which are even larger, afford the almost planar triselenasumanenes (SSe) (Supplementary Fig. 1). The shallower bowl depth of SS should facilitate the bowl-to-bowl inversion of the
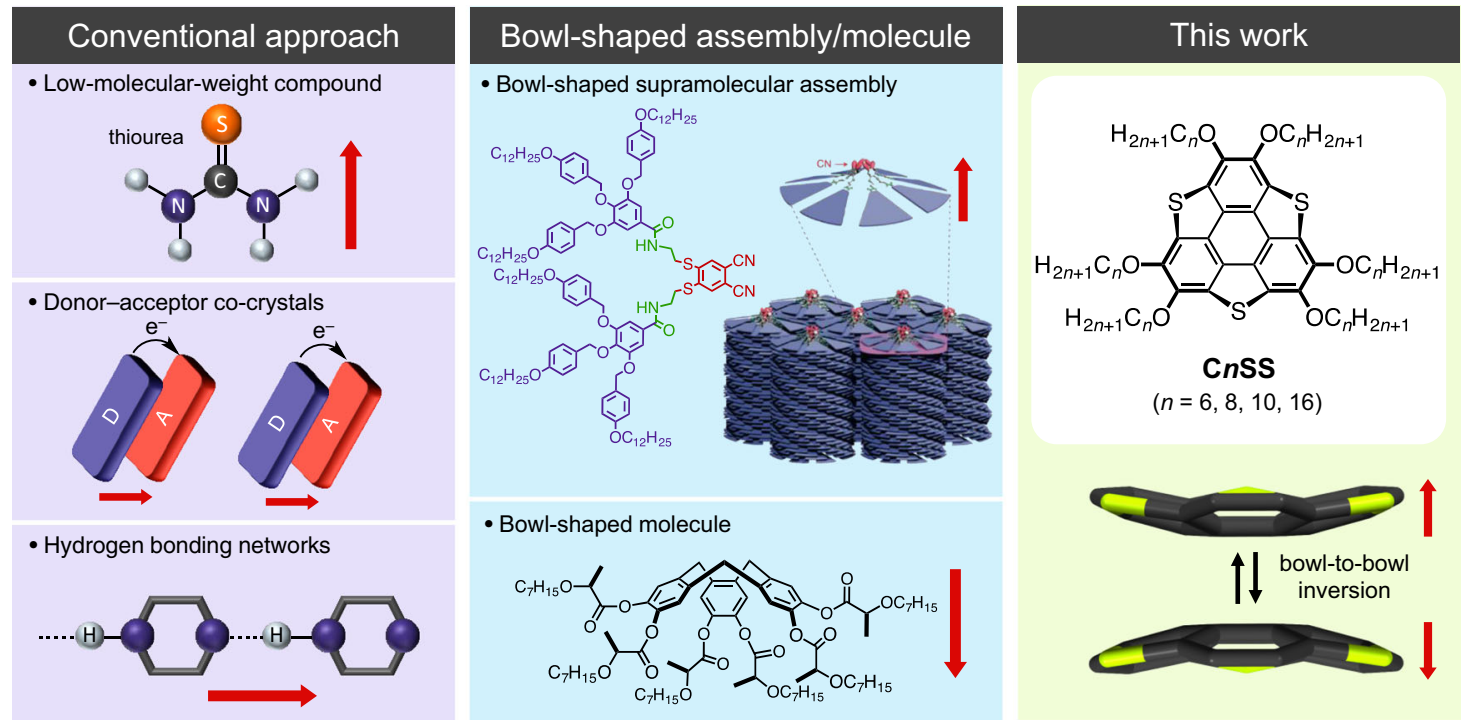

Fig. 1 Conventional approaches towards organic ferroelectric materials: anisotropic low-molecular-weight compounds, donor-acceptor-type chargetransfer complexes, and proton transfer in hydrogen-bonding networks (left). A ferroelectric bowl-shaped supramolecular assembly (reprinted from ref. ${ }^{3}$ ) and the chemical structure of a bowl-shaped molecule (middle). Chemical structures of CnSS molecules that contain a bowl-to-bowl invertible $\pi$-core (right). The red arrows indicate the dipole moment in each system. 


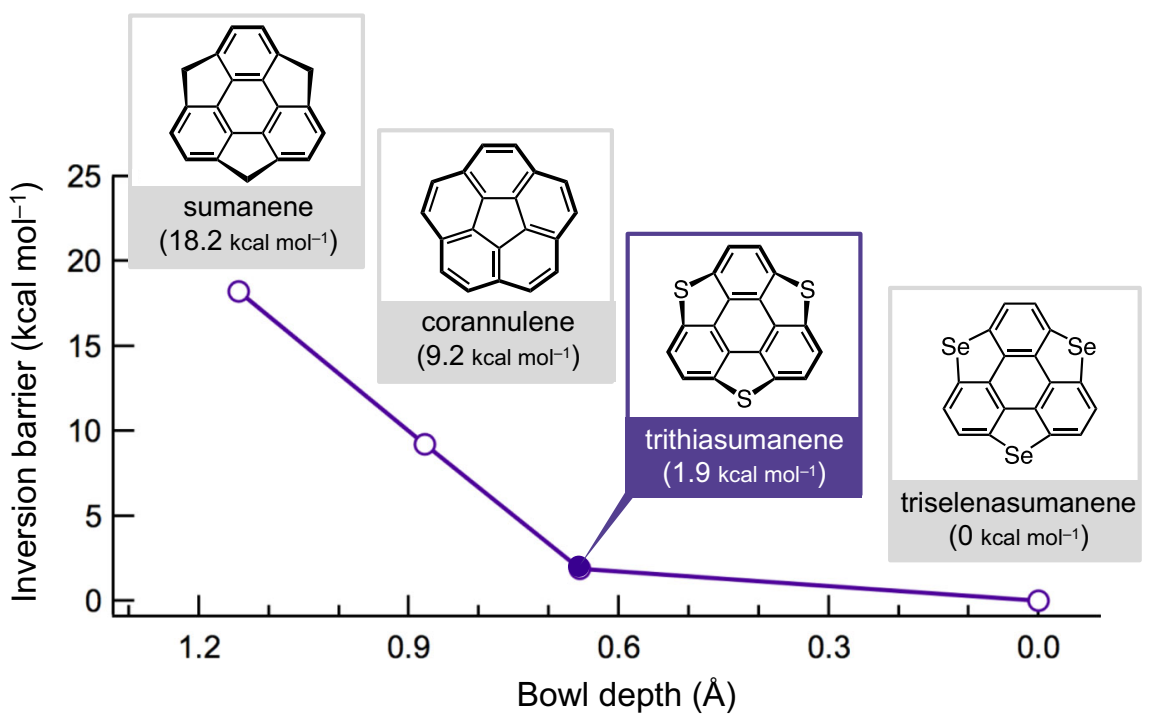

Fig. 2 Correlation between bowl depth and inversion barrier in bowl-shaped $\pi$-conjugated molecules.

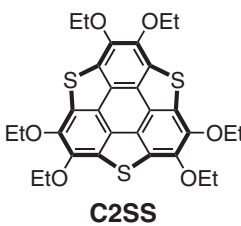

1) $\mathrm{BBr}_{3}$ (10 equiv.), $\mathrm{CH}_{2} \mathrm{Cl}_{2}$, $0{ }^{\circ} \mathrm{C}$ to rt 2) water C2SS
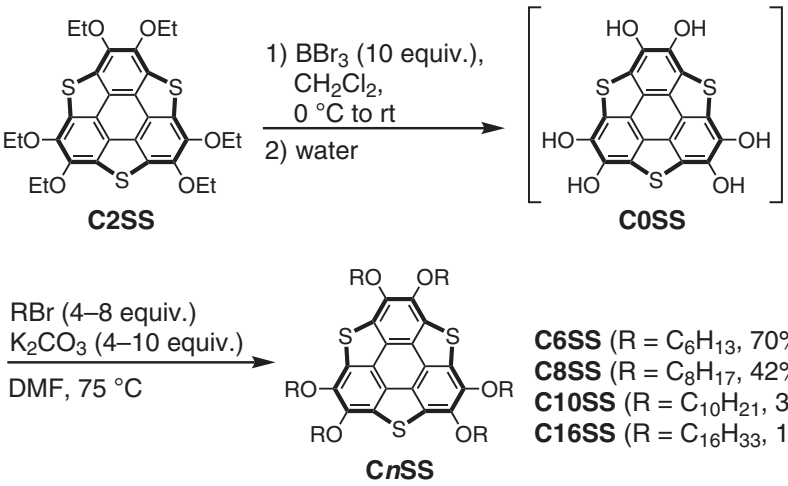

C6SS $\left(\mathrm{R}=\mathrm{C}_{6} \mathrm{H}_{13}, 70 \%\right)$ C8SS $\left(\mathrm{R}=\mathrm{C}_{8} \mathrm{H}_{17}, 42 \%\right)$ C10SS $\left(R=\mathrm{C}_{10} \mathrm{H}_{21}, 31 \%\right)$ C16SS $\left(\mathrm{R}=\mathrm{C}_{16} \mathrm{H}_{33}, 12 \%\right)$

Fig. 3 Synthesis of the targeted CnSS derivatives ( $n=6,8,10$, or 16). (a)

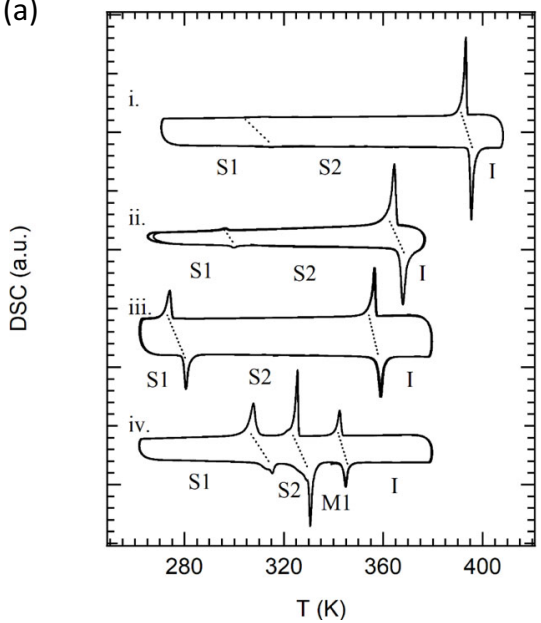

(b)

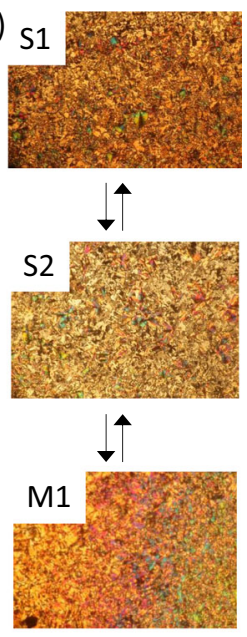

Fig. 4 Phase-transition behavior of CnSS derivatives ( $n=6,8$, 10, or 16). a DSC curves of (i) C6SS, (ii) C8SS, (iii) C10SS, and (iv) C16SS, where transformations between the $\mathrm{S} 1, \mathrm{~S} 2$, and $\mathrm{M} 1$ phases were reversible in the $T$-cycle. b POM images of C16SS at S1 ( $T=300 \mathrm{~K}), \mathrm{S} 2(T=320 \mathrm{~K})$, and M1 $(T=330 \mathrm{~K})$. aromatic core in the solid state upon applying an external stimulus. To promote motional freedom, as well as bowl inversion, six long alkoxy chains $\left(-\mathrm{OC}_{n} \mathrm{H}_{2 n+1}\right)$ were introduced in the $\mathbf{S S} \pi$ core, as such motional freedom should enhance the flexibility and internal thermal energy of the molecular assembly upon heating.

Herein, we report the synthesis of SS derivatives that bear $-\mathrm{OC}_{n} \mathrm{H}_{2 n+1}$ chains $(\mathbf{C} \boldsymbol{n S S} ; n=6,8,10$, or 16$)$ by dealkylation of C2SS, followed by re-alkylation of the obtained hexahydroxy intermediate COSS (Fig. 3). The starting material (C2SS) was prepared by a modified method for the synthesis of CASS $^{32}$. Treatment of C2SS with boron tribromide, followed by addition of water, afforded the air-sensitive hydroxy intermediate COSS, which was treated with the corresponding alkyl bromides $\left(\mathrm{C}_{n} \mathrm{H}_{2 n+1}-\mathrm{Br}\right.$; $n=6,8,10$, or 16) and potassium carbonate in dimethylformamide (DMF) to obtain the $\mathbf{C n S S}$ derivatives.

Phase-transition behavior and molecular assembly. The differential scanning calorimetry curves of $\mathbf{C n S S}(n=6,8,10$, or 16) in the solid state show a reversible phase transition (Fig. 4a). Crystals of C6SS, C8SS, and C10SS exhibit one reversible solid-solid (S1-S2) phase transition at 315, 299, and $280 \mathrm{~K}$ upon heating, and at 313,297 , and $274 \mathrm{~K}$ upon cooling, with melting points (S2-I phase transition) at 395,368 , and $359 \mathrm{~K}$, respectively (Table 1). The increasing length of the $-\mathrm{OC}_{n} \mathrm{H}_{2 n+1}$ chains results in lower S1-S2 and S2-I phase-transition temperatures. In polarized optical microscopy (POM) images, both the S1 and S2 phases exhibit birefringence under a cross-Nicol optical alignment in the absence of fluid behavior (top and middle POM images in Fig. 4b). On the contrary, fluid and birefringence properties were confirmed for the M1 phase of C16SS (bottom POM image in Fig. 4b), suggesting the formation of a liquid crystalline phase before melting. The M1-I phase-transition temperature and transition-enthalpy change $(\Delta H)$ for C16SS are $345 \mathrm{~K}$ and $15.1 \mathrm{~kJ} \mathrm{~mol}^{-1}$, respectively. The high structural flexibility of the $-\mathrm{OC}_{16} \mathrm{H}_{33}$ chains becomes apparent in the liquid crystalline phase above the S2 phase $(330-345 \mathrm{~K})$. The phasetransition behavior of the CnSS derivatives is very similar, except for the stabilization of the M1 phase for the C16SS derivative.

Next, we determined the molecular and assembly structure of CnSS ( $n=6,8,10$, or 16) by X-ray diffraction analyses. Although a single-crystal X-ray diffraction analysis was successfully carried 
Table 1 Summary of structural features, thermal properties, and P-E hysteresis of CnSS, CnSeS, and C6TP.

\begin{tabular}{|c|c|c|c|c|c|c|}
\hline \multirow[t]{2}{*}{ Compound } & \multirow[t]{2}{*}{ Structure of aromatic core } & \multicolumn{4}{|c|}{ Phase-transition and melting temperature ${ }^{d}(K)$} & \multirow[t]{2}{*}{ P-E response } \\
\hline & & S1-S2 & S2-M1 & S1- $\mathrm{Col}_{\mathrm{h}}$ & S2/M1/Col $=1$ & \\
\hline C6SS $^{a}$ & Bowl & 315 & - & - & 395 & Hysteresis \\
\hline C8SS & Bowl & 299 & - & - & 368 & Hysteresis \\
\hline C10ss ${ }^{b}$ & Bowl & 280 & - & - & 359 & Hysteresis \\
\hline C16SS ${ }^{\mathrm{C}}$ & Bowl & 315 & 330 & - & 345 & Hysteresis \\
\hline C4SeS $^{b}$ & Planar/bowld & 303 & - & - & 353 & Linear \\
\hline C6TPC & Planar & - & - & 343 & 371 & Linear \\
\hline
\end{tabular}

a Obtained at heating process of the DSC analysis.

bAbsense of fluid behavior in S1 and S2 phases.

cLiquid crystalline state at M1 or $\mathrm{Col}_{h}$ phase.

dStructure of triselenasumanene core of C4SeS obtained by crystallographic analysis is bowl-shaped, whereas the optimized structure obtained by the theoretical calculation is planar.

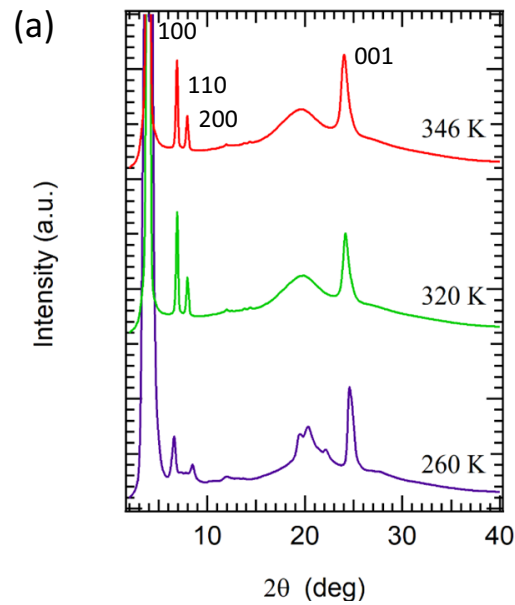

(b)

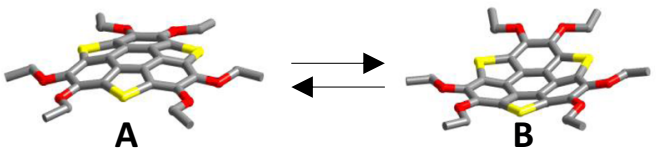

(c)

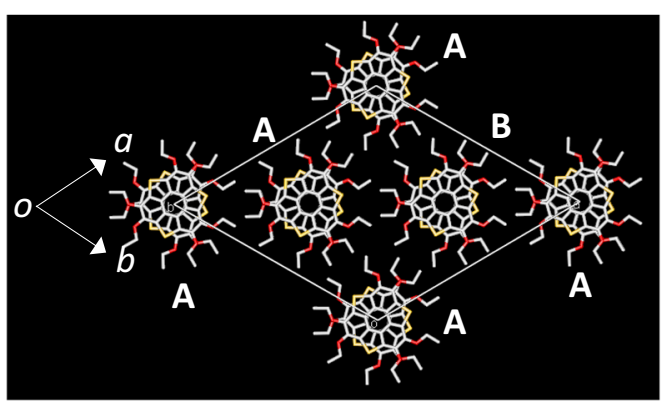

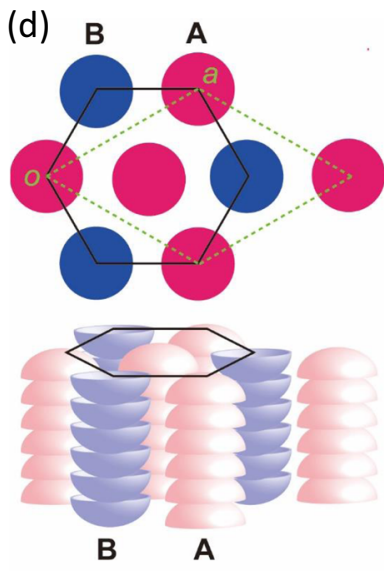

Fig. 5 Molecular assembly structure of CnSS. a PXRD patterns at $260 \mathrm{~K}$ (S1 phase), $320 \mathrm{~K}$ (S2 phase), and $346 \mathrm{~K}$ (S2 phase) with index assignments for the 100, 110, 200, and 001 reflections. b Bowl-to-bowl inversion from the A (up) to the B (down) conformation. c Unit cell of C2SS (viewed along the $c$-axis) based on the single-crystal $X$-ray diffraction analysis at $300 \mathrm{~K}$. The $A$ and $B$ columns coexist in the unit cell with an occupation ratio of $A / B=2 / 1$, suggesting a permanent dipole moment along the $c$-axis. $\mathbf{d}$ Schematic arrangement of the $A$ and $B$ columns in the hexagonal columnar (black solid lines) and trigonal (green dotted lines) lattices. The A and B columns are shown as pink and blue bowls, respectively.

out for C2SS at $300 \mathrm{~K}$, the single crystals obtained for the longchain derivatives $(n=6,8,10$, or 16) were unfortunately of insufficient quality. Thus, a temperature-dependent powder X-ray diffraction (PXRD) analysis was performed for each S1, S2, and M1 phase to obtain information on the molecular assembly structures. Figure $5 \mathrm{a}$ shows the temperature-dependent PXRD patterns for the $S 1 \quad(T=260 \mathrm{~K})$ and $\mathrm{S} 2(T=320$ and $346 \mathrm{~K})$ phases of C10SS. C6SS and C8SS exhibit almost identical PXRD patterns (Supplementary Fig. 2). The pattern of the S1 phase of C10SS (purple in Fig. 5a) is similar to that of the S2 phase. However, several sharp diffraction peaks at $2 \theta \sim 20^{\circ}$ broadened upon transition to the S2 phase (red and green in Fig. 5a), suggesting that the crystal symmetry of the latter is probably higher than that of the S1 phase due to thermally activated molecular motion. The most intense diffraction peak of the S2 phase at $2 \theta=3.960^{\circ}$ was assigned to the 100 reflection with a periodicity of $a=47.82 \AA$ for the trigonal (or hexagonal) crystal system, which corresponds to $a=25.4783(2) \AA$ for the singlecrystal X-ray diffraction analysis of C2SS with the polar space group $P 3 c 1$ at $300 \mathrm{~K}$. In addition, the two weak diffraction peaks at $2 \theta=6.908$ and $7.964^{\circ}$ were assigned to the 110 and 200 reflections, respectively, which is consistent with the formation of a hexagonal lattice. A linear relationship of the $h 00$ reflections was confirmed in the CnSS series $(n=2,6,8,10$, or 16)
(Supplementary Fig. 3), suggesting an identical packing structure for all CnSS derivatives. The broad reflection at $2 \theta \sim 20^{\circ}$ is probably due to the melting of the $-\mathrm{OC}_{n} \mathrm{H}_{2 n+1}$ chains, similar to the behavior in a discotic columnar liquid crystalline phase. It should be noted that the intense reflection at $2 \theta \sim 24.02^{\circ}$ corresponds to an average $\pi$-stacking distance of $3.71 \AA$.

A single-crystal X-ray diffraction analysis of C2SS at $300 \mathrm{~K}$ provided insight into the solid-state structure of C2SS, and the principal arrangements can potentially be extrapolated to the other $\mathbf{C}$ SS derivatives with longer alkyl chains. The molecular and packing structures for C2SS based on the single-crystal X-ray diffraction analysis are shown in Fig. 5b, c, respectively. A bowlshaped molecular structure was confirmed, where both the upbowl (A) and down-bowl (B) columns coexist in the unit cell. The $\pi$-stacking columns of A and B are hexagonally arranged with respect to each other (Fig. 5c). The occupation ratio of A-column/ B-column (2/1) in the unit cell is consistent with the formation of the polar space group P3c1. Figure $5 \mathrm{~d}$ shows the schematic arrangement of $\pi$-column A (red) and B (blue) in the trigonal (dashed green line) and hexagonal (solid black line) lattices. The polar space group $P 3 c 1$ of $\mathbf{C 2 S S}$ is consistent with a ferroelectric ground state along the $c$-axis, assuming bowl-to-bowl inversion between A and B. The PXRD patterns of the S1 and S2 phases for liquid crystalline C16SS are very similar, and that of the M1 
(a)

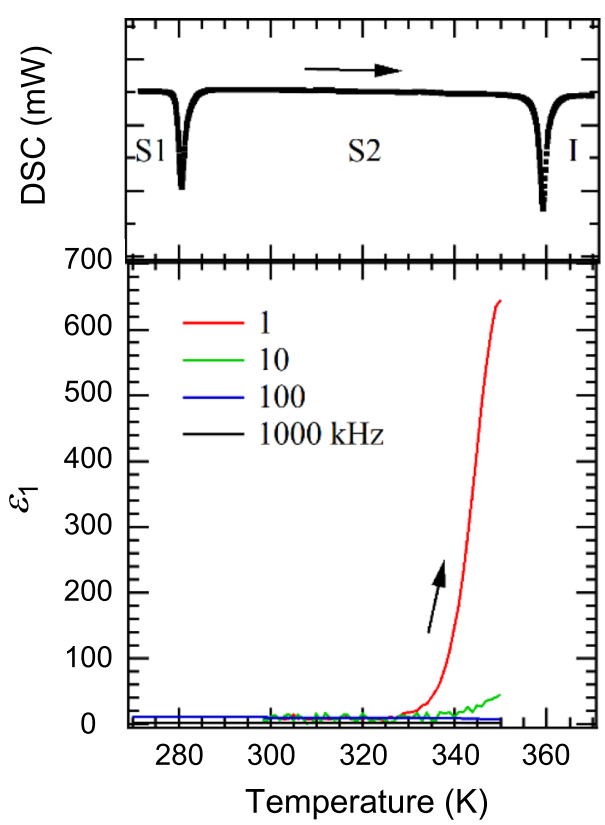

(c)

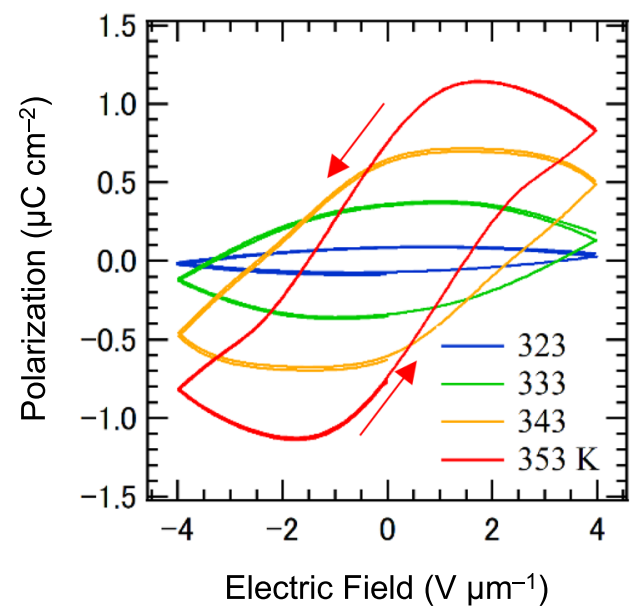

(b)

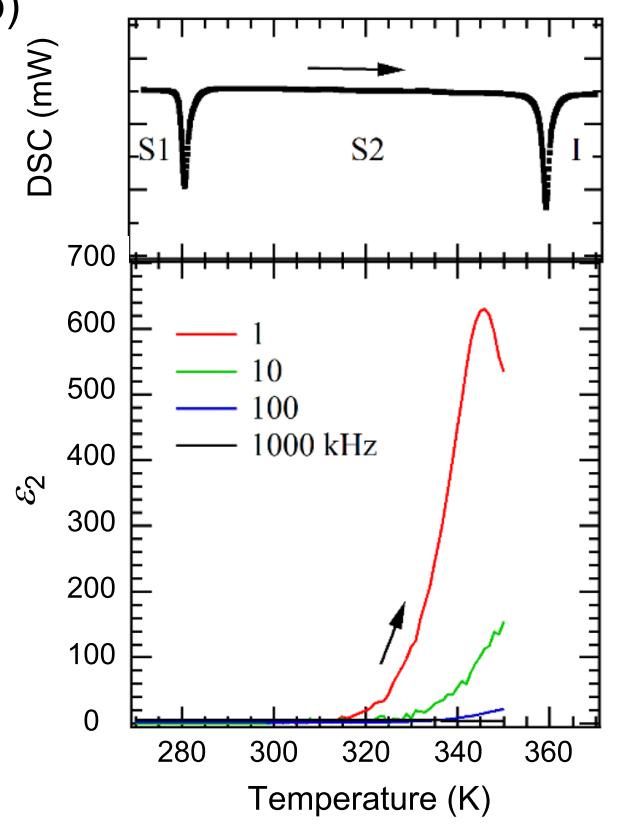

(d)

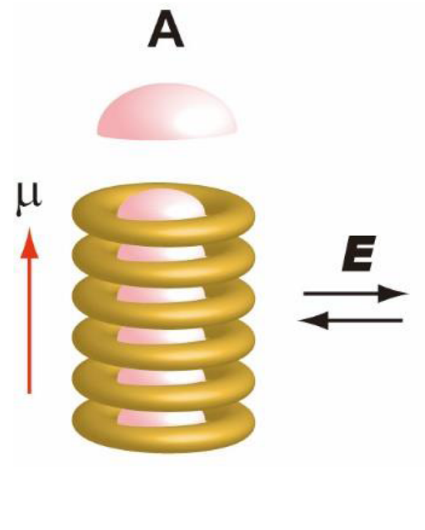

B

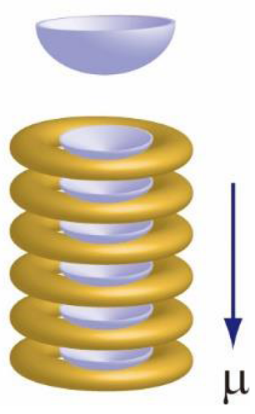

Fig. 6 Dielectric properties of C10SS. $T$ - and $f$-dependent a real $\varepsilon_{1}$ and $\mathbf{b}$ imaginary $\varepsilon_{2}$ components together with the DSC curves during the heating process. c $T$-dependent $P$-E hysteresis curves at $f=100 \mathrm{~Hz}$. d Schematic model of the bowl-to-bowl structural inversion upon application of an electric field, where molten $-\mathrm{OC}_{n} \mathrm{H}_{2 n+1}$ chains surround the polar bowl column.

phase is consistent with the formation of a discotic hexagonal columnar $\left(\mathrm{Col}_{\mathrm{h}}\right)$ liquid crystal phase (Supplementary Fig. $\left.4 \mathrm{~b}\right)$. In this $\mathrm{Col}_{\mathrm{h}}$ phase, the thermally activated melting of the six $-\mathrm{OC}_{16} \mathrm{H}_{33}$ chains and the free rotation of the $\pi$-stacking columns along the orientation of the director occur simultaneously, weakening the intensity of the broad 001 reflection due to thermal fluctuation of the $\pi$-stacking $d_{001}$-spacing. Although the $\mathrm{Col}_{\mathrm{h}}$ mesophase was not observed for C6SS, C8SS, and C10SS, the molten state of the six $-\mathrm{OC}_{n} \mathrm{H}_{2 n+1}$ chains was confirmed by the broad diffraction at $2 \theta \sim 20^{\circ}$ (Supplementary Fig. 2). The inplane rotation of each bowl-shaped molecule is likely suppressed in the $\pi$-stacking columns of the S2 phase, also disappearing in the $\mathrm{Col}_{\mathrm{h}}$ phase for certain $\mathrm{CnSS}(n=2,6,8$, or 10$)$. The thermally activated molten state of the $-\mathrm{OC}_{n} \mathrm{H}_{2 n+1}$ chains plays an important role in the phase transitions and in the ferroelectric response of such bowl-shaped CnSS.

Ferroelectric response. Dielectric features arise from the molecular motion of polar structural units within a molecular assembly. In the present $\mathbf{C n S S}$ derivatives, such polar structural units are only observed in the bowl-shaped $\pi$-plane, whose $\pi$ stacking generates a macro dipole moment along the $\pi$-stacked column. The $\pi$-stacked columns of $\mathrm{CnSS}$ in high-temperature S2 phase were easily oriented along the direction normal to the ITO surface after the cooling from isotropic liquid, which was confirmed in the dark POM images of the homeotropic orientation on the substrate surface (Supplementary Fig. 5). A polar crystal structure with space group $P 3 c 1$ was identified for a single crystal of C2SS, where the occupation number of the polar A and B $\pi$ stacking bowls was not balanced, producing a ferroelectric dipole ground state. The second-harmonic generation (SHG) activity was observed at C2SS at $298 \mathrm{~K}$, in which the magnitude was similar to that of sucrose (Supplementary Figs. 6-7). Figure $6 a, b$ shows the temperature $(T)$ - and frequency $(f)$-dependent real $\left(\varepsilon_{1}\right)$ and imaginary $\left(\varepsilon_{2}\right)$ components of the dielectric constants of C10SS using a sandwich-type electrode. The $T$ - and $f$-dependent $\varepsilon_{1}$ responses around $300 \mathrm{~K}$ increased monotonically with increasing temperature and frequency above $330 \mathrm{~K}$ for the S2 phase. Dielectric anomalies were not observed around the S1-S2 phase transition. On the contrary, the $\varepsilon_{2}-T$ plots at $f=1 \mathrm{kHz}$ 
showed a dielectric peak at $349 \mathrm{~K}$, i.e., a lower temperature than that of the S2-I phase transition $(356 \mathrm{~K})$. Almost the same $f$ - and T-dependent dielectric constants were obtained for C6SS and C8SS (Supplementary Fig. 8). Interestingly, a ferroelectric polarization-electric field $(P-E)$ hysteresis at $f=100 \mathrm{~Hz}$ for C10SS was confirmed in the high-temperature S2 phase (Fig. 6c), i.e., it was clearly observed at 353 and $343 \mathrm{~K}$ with a remanent polarization $\left(P_{\mathrm{r}}=0.5-0.7 \mu \mathrm{Ccm}^{-2}\right)$ and coercive voltage $\left(E_{\mathrm{th}} \approx\right.$ $\left.1.5 \mathrm{~V} \mu \mathrm{m}^{-1}\right)$. Time-dependent polarization behavior of C10SS at $343 \mathrm{~K}$ showed that the polarization was retained for $4000 \mathrm{~ms}$ after applying a pulse voltage (Supplementary Fig. 9). The appearance of an $\varepsilon_{2}$ peak at $356 \mathrm{~K}$ and $f=100 \mathrm{~Hz}$ is consistent with the thermally activated molecular motion of the polar structural units at $\sim 100 \mathrm{~Hz}$ and $356 \mathrm{~K}$, corresponding to a structural inversion of the $\pi$-stacking columns (Fig. $6 \mathrm{~d}$ ). The observed $P-E$ hysteresis curve is explained by the polling process between the electrodes, which orients each column and domain along the sandwich direction. The homeotropic orientation of each $\pi$-stacking column aligns the direction of macro dipole moment normal to the ITO surface without the polling process. The bowl-to-bowl structural inversion is thermally activated even in the solid S2 phase. The thermal melting of the six $-\mathrm{OC}_{n} \mathrm{H}_{2 n+1}$ chains around the polar $\pi$-stacking column plays an essential role in the dipole inversion of each polar column, resulting in ferroelectric dipole relaxation via structural bowl-to-bowl inversion. Similar ferroelectric relaxation processes were observed in the S2 phase of C6SS and C8SS (Supplementary Fig. 10), whereas liquid crystalline C16SS also exhibited ferroelectricity in the M1 phase

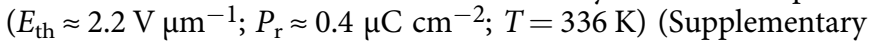
Fig. 11).

\section{Discussion}

The shallower bowl depth of the SS $\pi$-core is crucial for the bowl inversion in the solid state. The dipole moment $(\mu)$ along the bowl-shaped $\pi$-plane of SS is $1.03 \mathrm{D}^{25}$. The theoretically calculated bowl-to-bowl inversion energy $\left(\Delta E_{\mathrm{b}}\right)$ in the gas phase is relatively low $\left(1.9 \mathrm{kcal} \mathrm{mol}^{-1}\right)$, i.e., by one order of magnitude lower than that of sumanene $\left(\Delta E_{\mathrm{b}}=18.2 \mathrm{kcal} \mathrm{mol}^{-1}\right)^{25}$ (Fig. 2), but higher than the room temperature energy $\left(\mathrm{k}_{\mathrm{B}} T \approx 0.58 \mathrm{kcal}\right.$ $\left.\mathrm{mol}^{-1}\right)$. The bowl-to-bowl inversion of $\mathrm{CnSS}(n=6,8,10$, or 16$)$ was observed at $\sim 350 \mathrm{~K}\left(\sim 0.7 \mathrm{kcal} \mathrm{mol}^{-1}\right)$ in the $\pi$-stacked onedimensional molecular assembly, whereas such an inversion could not be observed in the liquid crystalline phase of a $-\mathrm{SC}_{n} \mathrm{H}_{2 n+1}$-substituted sumanene derivative ${ }^{19}$. The small curvature angle of the $\pi$-plane of SS leads to a smaller $\Delta E_{\mathrm{b}}$, thus enabling the bowl-to-bowl inversion even in the solid state, where the thermal melting of the alkyl chains serves as an entropy source for the polar $\pi$-stacking structure.

In order to assess the correlation between the ferroelectricity and the molecular structure, the shallow bowl-shaped selenium derivative C4SeS was prepared by replacement of three $\mathrm{S}$ atoms by $\mathrm{Se}^{32}$. Moreover, the $\pi$-planar triphenylene derivative C6TP was evaluated in terms of its phase transitions, $T$ - and $f$-dependent dielectric constants, and $P-E$ hysteresis curves. Although the theoretically obtained structure of triselenasumanene (SeS) is planar in the absence of alkoxy groups (Fig. 2), the structure of C4SeS presents a shallow bowl shape with bowl depths ranging from $0.32 \AA$ to $0.46 \AA$, as determined by a single-crystal X-ray diffraction analysis ${ }^{32}$. Crystals of $\mathbf{C 4 S e S}$ exhibit a phase transition from crystalline solid (S1) to isotropic liquid (IL) at $358 \mathrm{~K}$ in the absence of the intermediate thermally activated S2 and/or $\mathrm{Col}_{h}$ liquid crystalline phases found for the CnSS derivatives (Supplementary Fig. 12a). Single crystals of C4SeS with polar space group $R 3$ present one-dimensional polar bowl-to-bowl $\pi$-stacking columns along the $c$-axis, where the macroscopic dipole moment
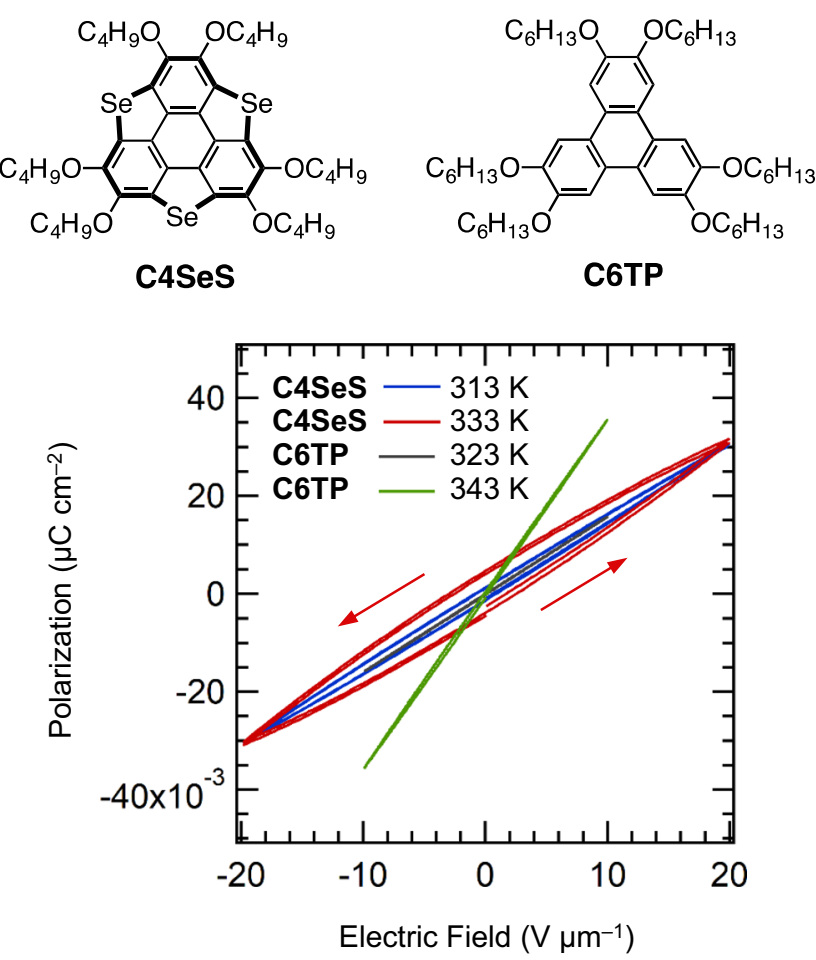

Fig. 7 Molecular structures of C4SeS and C6TP (top) and T-dependent $P-E$ hysteresis curves at $f=100 \mathrm{~Hz}$ (bottom).

may potentially exhibit dipole inversion and ferroelectricity upon application of an external electric field, similar to the case of the CnSS crystals. It should be noted here that the crystalline S1 state of C4SeS does not contain thermally molten $-\mathrm{OC}_{n} \mathrm{H}_{2 n+1}$ chains. The temperature-dependent PXRD patterns of C4SeS in the temperature range from 150 to $350 \mathrm{~K}$ show a highly crystalline phase with sharp diffraction peaks and the absence of a broad diffraction at $2 \theta \sim 20^{\circ}$, which suggests a non-thermally activated molten state of the $-\mathrm{OC}_{4} \mathrm{H}_{9}$ chains (Supplementary Fig. 12b). Furthermore, dielectric anomalies were not observed for the $T$ and $f$-dependent $\varepsilon_{1}$-values, which is consistent with the absence of a bowl-to-bowl inversion in the crystalline S1 state (Supplementary Fig. 13). The P-E curves of the S1 phase of C4SeS at 313 and $333 \mathrm{~K}$ revealed a linear behavior at $f=100 \mathrm{~Hz}$, which is consistent with a paraelectric ground state (Fig. 7). Although a polar space group was observed for crystalline C4SeS, its highly crystalline and rigid molecular assembly structure suppresses the thermally activated bowl-to-bowl inversion. On the contrary, the $\pi$-planar molecule C6TP exhibits an $\mathrm{S} 1-\mathrm{Col}_{h}$ phase transition at $343 \mathrm{~K}$ and $\mathrm{a} \mathrm{Col}_{\mathrm{h}}-\mathrm{I}$ transition at $371 \mathrm{~K}$, where a thermally stable columnar liquid crystalline phase was observed within a temperature range of $\sim 30 \mathrm{~K}$ (Supplementary Fig. 14a). However, both the $T$ - and $f$-dependent $\varepsilon_{1}$ behavior of C6TP did not show any evidence for bowl-to-bowl inversion or thermally activated molecular motion of the polar structural units within the molecular assembly (Supplementary Fig. 14b, c). Stacked structures of $\pi$-planar molecules do not exhibit a macroscale dipole moment. Therefore, linear $P-E$ hysteresis curves were obtained both in the $\mathrm{S} 1$ and $\mathrm{Col}_{\mathrm{h}}$ phases due to the absence of dipole inversion, despite the flexibility of the $\mathrm{Col}_{\mathrm{h}}$ phase. Therefore, thermally activated melting and/or a dynamic state of the peripheral $-\mathrm{OC}_{n} \mathrm{H}_{2 n+1}$ chains and the bowl-to-bowl inversion at the central SS core are both essential for the ferroelectricity exhibited by the CnSS derivatives in the $\mathrm{S} 2$ and $\mathrm{Col}_{\mathrm{h}}$ phases. 
To elucidate the ferroelectric mechanism of CnSS, the dipole moment and $P_{\mathrm{r}}$ values were determined by DFT calculations at the B3LYP 6-31 G(d,p) level of theory on model compounds SS and C1SS. The macroscopic polarization, $P_{\mathrm{r}}$, was obtained from Eq. (1):

$$
P_{\mathrm{r}}=\frac{\sum_{i=1}^{n} P_{i}}{\Delta V}=\frac{\Sigma p_{\mathrm{mol}}}{\Delta V}
$$

(a)
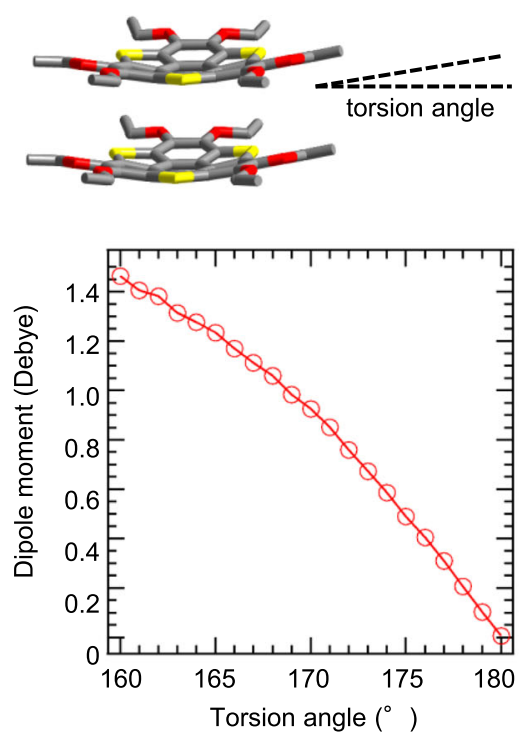

(c)

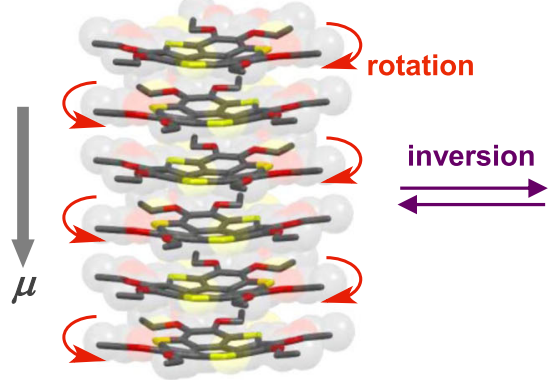

(d)

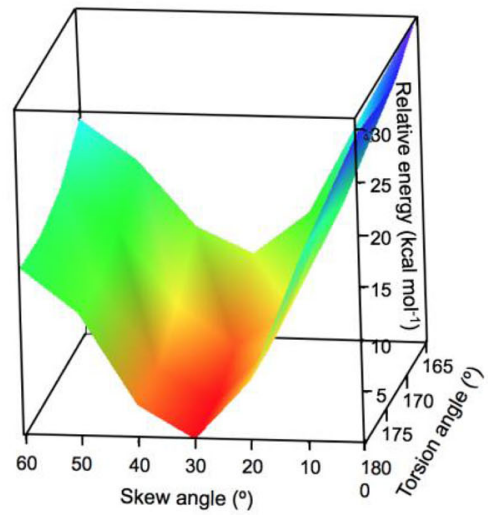

The calculated dipole moment along the normal to the bowlshaped $\pi$-plane of the SS molecule $(\mu=0.84 \mathrm{D})$ afforded a theoretical $P_{\mathrm{r}}$ value of $0.19 \mu \mathrm{C} \mathrm{cm}^{-2}$ for $\mathrm{CnSS}$ at $343 \mathrm{~K}$, which is inconsistent with the experimental value $\left(P_{\mathrm{r}} \approx 0.6 \mu \mathrm{C} \mathrm{cm}^{-2}\right)$. However, collective motion of dipole moment in the $\pi$-stacking column effectively enhanced the macroscale dipole moment and the magnitude of the polarization ${ }^{33,34}$. Although the SHG activity was clearly confirmed in highly crystalline C2SS with polar space (b)
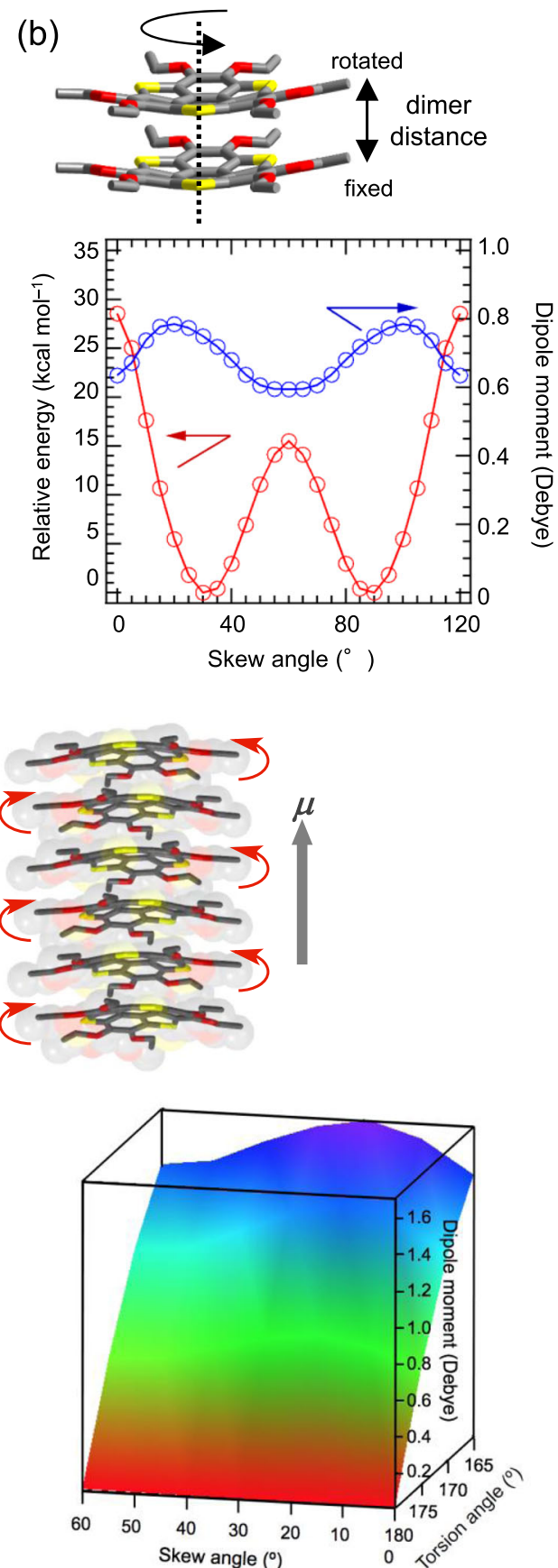

Fig. 8 Ferroelectric relaxation mechanism of CnSS derivative. a Relative energy (red in left scale) and dipole moment (blue in right scale) vs. torsion angel plots of dimeric (C1SS) 2 based on DFT calculations at the B3LYP 6-31 G(d) level of theory. The interdimer distance and skew angle between central benzene rings were fixed with those of C2SS dimer in the crystal. The smallest total energy of $\pi$-planar structure with a dihedral angle of $180^{\circ}$ was defined as zero. b Relative energy (red in left scale) and dipole moment (blue in right scale) vs. skewed angel plots of dimeric (C1SS) 2 . The total energy of bowlshaped structure with a skew angle of $30^{\circ}$ and $90^{\circ}$ was defined as zero. c Schematic dipole inversion mechanism coupled with fluctuation/inversion of bowl-shaped conformation and skew rotation. $\mathbf{d}$ 3D plots of relative energy (left) and dipole moment (right) against torsion angle and skew angle of dimeric (C1SS) $)_{2}$. 
group of $P 3 c 1$, there were insufficient SHG signals for the other ferroelectric CnSS derivatives at high-temperature S2 phase. The SHG activity should be observed in the ferroelectric ground state due to the polar space group, while highly viscous plastic crystalline S2 phase with thermally melting state of six $-\mathrm{OC}_{n} \mathrm{H}_{2 n+1}$ chains exhibited the weak SHG activity in spite of their $P-E$ hysteresis. To discuss this ferroelectric relaxation behavior of CnSS derivatives, we estimated a possible polarization mechanism for dipole inversion and relaxation process of the bowl-tobowl inversion based on the theoretical DFT calculation using a model compound of C1SS dimer (Fig. 8), where the interdimer distance and skew angle between central benzene rings were fixed at the same as those observed in the C2SS single-crystal X-ray analysis. The bowl-depth (torsion angle at C-C-C-S bonds) was increased in a stepwise manner from the $\pi$-planar structure (torsion angle $=180^{\circ}$ ) to the bowl-shaped one (torsion angle $=$ $160^{\circ}$ ) of C1SS dimer (Fig. 8a). Thermally fluctuated structural transformation between the $\pi$-planar and bowl-shaped conformations at plastic crystalline S2 phase easily activated dipole moment from $0 \mathrm{D}$ at $\pi$-planar structure to $1.46 \mathrm{D}$ at bowl-shaped one by the increase in the torsion angle (Fig. 8a). The intermolecular interaction between the $\pi$-stacking columns was dominated by the hydrophobic interaction between the alkyl chains, and consequently thermal melting decreased the magnitude of intermolecular interaction and activated the bowl-to-bowl inversion in the column. Therefore, the thermally activated structural fluctuation between the polar and the non-polar molecular conformations in the 1D $\pi$-stacking column should be easily activated in highly temperature viscous S2 phase, where the polarity and SHG activity were thermally disturbed in bulk due to the thermal fluctuation.

The second important molecular motion is a relative skew rotation of C1SS dimer, where the rotation angle of the upper C1SS relative to the lower one changed the magnitude of the relative energy at fixed bowl-shaped conformation with the fixed torsion angle of $175^{\circ}$ (Fig. 8b). When the bowl-shaped C1SS dimer was directly overlapped with a translational motion along the $\pi$-dimer with the sterically repulsive S-S interactions along the $\pi$-stack, a large relative energy around $30 \mathrm{kcal} \mathrm{mol}^{-1}$ was observed as a forbidden in-plane free rotational motion. The energy minima for the skew angle rotation were observed at the angles of $30^{\circ}$ and $90^{\circ}$ with every $60^{\circ}$ rotation of the upper C1SS, while the maxima of the dipole moment of $0.84 \mathrm{D}$ were also observed around 20 and $100^{\circ}$. The forbidden in-plane free rotation inhibited the formation of $\mathrm{Col}_{\mathrm{h}}$ liquid crystal phase. The restricted $30^{\circ}$-rotated $\pi$-stacking interaction of C1SS in the $\pi$ stacking column was also thermally fluctuated in the hightemperature viscous S2 phase, where the motion was also coupled with the inversion motion of bowl-shaped conformation via the $\pi$-planar one (Fig. 8c). Although the $\pi$-stacking distance in the $\pi$ dimer (C1SS $)_{2}$ was associated with the relative energy, it did not affect the theoretical dipole moment (Supplementary Fig. 15). Three kinds of motional freedoms of torsion, skew, and dimer separation distance were activated at S2 phase, among which the first two coupled to generate the macroscale dipole moment under the applied electric field (Fig. 8d). When the electric field was applied to the thermally fluctuated $\pi$-stacking column of $(\mathbf{C n S S})_{\infty}$, the polarized molecular conformation in the 1D assembly should be stabilized at the bowl-shaped and twisted $\pi$ stacking columnar structure keeping the maximum dipole structure. Such polar molecular assembly was thermally relaxed after the removal of the outer electric field, which resulted in the ferroelectric $P-E$ hysteresis and relaxation behavior at hightemperature viscous S2 phase. In the order-disorder type molecular ferroelectrics, the low-frequency response at $f=0.1-10 \mathrm{~Hz}$ has been typically observed in the $P-E$ hysteresis curve. However, the relatively high-frequency responses of the $P-E$ hysteresis curve at $f=100-200 \mathrm{~Hz}$ for $\mathrm{CnSS}$ derivatives were consistent with the structural relaxation process from the polar molecular assembly structure under the applied electric field.

In summary, the collective dipole inversion of $1 \mathrm{D} \pi$-stacking bowl-shaped molecules showed the ferroelectric response due to bowl-to-bowl inversion, where the chemical design of the bowldepth $\left(\mathrm{CH}_{2} \rightarrow \mathrm{S} \rightarrow \mathrm{Se}\right)$ tuned the suitable inversion barrier in bulk and possible dipole inversion by the application of the electric field. CnSS derivatives indicated the phase transition to high viscous S2 phase, where complete melting state of alkyl chains formed the 1D plastic crystalline phase. The thermally activated highly melting state enabled collective bowl-to-bowl inversion associated with the in-plane rotation stabilized by S•••S contacts and dipole-dipole interactions. The $P-E$ hysteresis responses were adequately high even in dynamic molecular system, caused by the high-speed relaxation of the polar bowlstacking $\pi$-column under the electric field. The present finding reveals that the wide variation of molecular designs for organic $\pi$ molecules enables us to fabricate flexible and high-density organic memory devices.

\section{Methods}

Materials. C6TP and C4SeS were prepared following reported methods ${ }^{32,35}$. C2SS was synthesized by a modified method for the synthesis of other trichalcogenasumanene derivatives (Supplementary Information, section 2$)^{32}$. CnSS $(n=6,8$, 10 , or 16) were prepared by the following general procedure: a dichloromethane solution of boron tribromide $\left(1.0 \mathrm{~mol} \mathrm{~L}^{-1}, 10\right.$ equiv. $)$ was slowly added to a dichloromethane solution of $\mathbf{C 2 S S}$ at $0{ }^{\circ} \mathrm{C}$, before the mixture was stirred at room temperature for $2 \mathrm{~h}$. Then, water was slowly added to the reaction mixture at $0{ }^{\circ} \mathrm{C}$. The resulting solid was collected by filtration and washed with dichloromethane and water to furnish intermediate COSS. The obtained solid was dissolved in DMF, and potassium carbonate (4-10 equiv.) and the corresponding alkyl bromide (4-8 equiv.) were added to the solution. The mixture was stirred overnight at $75^{\circ} \mathrm{C}$. After cooling to room temperature, the mixture was filtered to remove an insoluble solid. Water was added to the obtained solution, and the organic layer was extracted with a mixture of ethyl acetate and hexane $(4: 1, \mathrm{v} / \mathrm{v})$. The combined organic extracts were dried over anhydrous magnesium sulfate before the inorganic solids were removed by filtration. After evaporation of all volatiles, the crude products were purified by column chromatography on silica gel (eluent: hexane/ dichloromethane) to afford CnSS.

\begin{abstract}
Measurements. Temperature-dependent crystallographic data for a single crystal of C2SS at $300 \mathrm{~K}$ were collected on a Rigaku RAPID-II diffractometer equipped with a rotating anode and a multilayer confocal optic using graphitemonochromated $\mathrm{Cu}-\mathrm{Ka}$ radiation $(\lambda=1.54187 \AA)$. Structural refinements were carried out using the full-matrix least-squares method on $F^{2}$. Temperaturedependent PXRD measurements were performed on a Rigaku SmartLab diffractometer using $\mathrm{Cu}-\mathrm{Ka}$ radiation $(\lambda=1.54187 \AA)$. Temperature-dependent dielectric constants were determined by the two-probe AC impedance method at frequencies from $1 \mathrm{kHz}$ to $1 \mathrm{MHz}$ (Hewlett-Packard, HP4194A) with a liquid crystal cell placed in a temperature control system (Linkam, LTS350). The $P-E$ curves were measured with a commercially available ferroelectric tester (Precision LC, Radient Technologies) using a liquid crystalline cell with an electrode gap of 2 $\mu \mathrm{m}$. The CnSS derivatives in the liquid state were introduced in the electrode gap of the liquid crystal cell.
\end{abstract}

\section{Data availability}

All the data generated or analyzed during this study are included in this published article (and its supplementary information files) or available from the authors upon reasonable request. The crystallographic information for this paper has been deposited at The Cambridge Crystallographic Data Center (http://www.ccdc.cam.ac.uk) under reference number CCDC-1912824

Received: 24 May 2019; Accepted: 8 January 2021; Published online: 03 February 2021

\section{References}

1. Lines, M. E. \& Glass, A. M. Principles and applications of ferroelectrics and related materials (Oxford University Press, New York, 1977). 
2. Horiuchi, S. \& Tokura, Y. Organic ferroelectrics. Nat. Mater. 7, 357-366 (2008).

3. Tayi, A. S., Kaeser, A., Matsumoto, M., Aida, T. \& Stupp, S. I. Supramolecular ferroelectrics. Nat. Chem. 7, 281-294 (2015).

4. Solomon, A. L. Thiourea, a new ferroelectric. Phys. Rev. 104, 1191 (1956).

5. Goldsmith, G. J. \& White, J. G. Ferroelectric behavior of Thiourea. J. Chem. Phys. 31, 1175-1187 (1959).

6. Harada, J. et al. Directionally tunable and mechanically deformable ferroelectric crystals from rotating polar globular ionic molecules. Nat. Chem. 8, 946-952 (2016).

7. Tokura, Y. et al. Domain-well dynamics in organic charge-transfer compounds with one-dimensional ferroelectricity. Phys. Rev. Lett. 63, 2405-2408 (1989).

8. Kagawa, F. et al. Electric-field control of solitons in a ferroelectric organic charge-transfer salt. Phys. Rev. Lett. 104, 227602-227604 (2010).

9. Tayi, A. S. et al. Room-temperature ferroelectricity in supramolecular networks of charge-transfer complexes. Nature 488, 485-489 (2012).

10. Horiuchi, S. et al. Above-room-temperature ferroelectricity in a singlecomponent molecular crystal. Nature 463, 789-792 (2010)

11. Horiuchi, S. et al. Above-room-temperature ferroelectricity and antiferroelectricity in benzimidazoles. Nat. Commun. 3, 1308 (2012).

12. Owczarek, M. et al. Flexible ferroelectric organic crystals. Nat. Commun. 7, 13108 (2016)

13. Lunkenheimer, P. et al. Ferroelectric properties of charge-ordered $\alpha$-(BEDTTTF)2I3. Phys. Rev. B 91, 245132-1-7 (2015).

14. Akutagawa, T. et al. Ferroelectricity and polarity control in solid state flip-flop supramolecular rotators. Nat. Mater. 8, 342-347 (2009).

15. Miyajima, D. et al. Ferroelectric columnar liquid crystal featuring confined polar groups within core-shell architecture. Science 336, 209-213 (2012).

16. Sato, K., Itoh, Y. \& Aida, T. Columnarly assembled liquid-crystalline peptidic macrocycles unidirectionally orientable over a large area by an electric field. $J$. Am. Chem. Soc. 133, 13767-13769 (2011).

17. Malthete, J. \& Collet, A. Inversion of the cyclotribenzylene cone in a columnar mesophase: a potential way to ferroelectric materials. J. Am. Chem. Soc. 109, 7544-7545 (1987).

18. Miyajima, D. et al. Liquid crystalline corannulene responsive to electric field. J. Am. Chem. Soc. 131, 44-45 (2009).

19. Shoji, Y. et al. Hexathioalkyl sumanenes: an electron-donating buckybowl as a building block for supramolecular materials. Chem. Sci. 8, 8405-8410 (2017).

20. Guilleme, J. et al. A columnar liquid crystal with permanent polar order. J. Mater. Chem. C. 3, 985-989 (2015).

21. Guilleme, J. et al. Polar switching in a lyotropic columnar nematic liquid crystal made of bowl-shaped molecules. Adv. Mater. 27, 4280-4184 (2015).

22. García-Iglesias, M. et al. A versatile method for the preparation of ferroelectric supramolecular materials via radical end-functionalization of vinylidene fluoride oligomers. J. Am. Chem. Soc. 138, 6217-6223 (2016).

23. Gorbunov, A. V. et al. Ferroelectric self-assembled molecular materials showing both rectifying and switchable conductivity. Sci. Adv. 3, e1701017 (2017).

24. Imamura, K., Takimiya, K., Otsubo, T. \& Aso, Y. Triphenyleno[1,12-bcd:4,5-b $\left.{ }^{\prime} \mathrm{c}^{\prime} \mathrm{d}^{\prime}: 8,9-\mathrm{b}^{\prime \prime} \mathrm{c}^{\prime \prime} \mathrm{d}^{\prime \prime}\right]$ trithiophene: the first bowl-shaped heteroaromatic. Chem. Commun. Issue 18, 1859-1860 (1999).

25. Priyakumar, U. D. \& Sastry, G. N. Heterobuckybowls: a theoretical study on the structure, bowl-to-bowl Inversion barrier, bond length alternation, structure-inversion barrier relationship, stability, and synthetic feasibility. $J$. Org. Chem. 66, 6523-6530 (2001).

26. Seiders, T. J., Baldridge, K. K., Grube, G. H. \& Siegel, J. S. Structure/energy correlation of bowl depth and inversion barrier in corannulene derivatives: Combined experimental and quantum mechanical analysis. J. Am. Chem. Soc. 123, 517-525 (2001).

27. Saito, M., Furukawa, S., Kobayashi, J. \& Kawashima, T. The chemistry of heterasumanenes. Chem. Rec. 16, 64-72 (2016).

28. Li, X. \& Shao, X. Synthesis of heterasumanene. Synlett 25, 1795-1798 (2014).

29. Saito, M., Shinokubo, H. \& Sakurai, H. Figuration of bowl-shaped $\pi-$ conjugated molecules: properties and functions. Mater. Chem. Front. 2, 1-27 (2018).

30. Furukawa, S., Kobayashi, J. \& Kawashima, T. Development of a sila-Friedel -Crafts reaction and its application to the synthesis of dibenzosilole derivatives. J. Am. Chem. Soc. 131, 14192-14193 (2009).
31. Furukawa, S. et al. Triphosphasumanene trisulfide: High out-of-plane anisotropy and Janus-type $\pi$-surfaces. J. Am. Chem. Soc. 139, 5787-5792 (2017).

32. Li, X. et al. Non-pyrolytic, large-scale synthesis of trichalcogenasumanene: a two-step approach. Angew. Chem. Int. Ed. 53, 535-538 (2013).

33. Louis, L. et al. Polarization canting in ferroelectric diisopropylammoniumhalide molecular crystals: a computational first principles study. J. Mater. Chem. C. 6, 1143-1152 (2018).

34. Horiuchi, S., Kobayashi, K., Kumai, R. \& Ishibashi, S. Proton tautomerism for strong polarization switching. Nat. Commun. 8, 14426 (2017).

35. Gupta, M. et al. A new strategy towards the synthesis of a room-temperature discotic nematic liquid crystal employing triphenylene and pentaalkynylbenzene units. Chem. Commun. 53, 3014-3017 (2017).

\section{Acknowledgements}

This work was supported by a Grant-in-Aid for Scientific Research on Innovative Areas " $\pi$-System Figuration: Control of Electron and Structural Dynamism for Innovative Functions" from the Japan Society for the Promotion of Science (JSPS) (JP26102006 to M.S. and JP26102007 to T.A.) and "Stimuli-responsive Chemical Species for the Creation of Functional Molecules" (15H00918 to S.F.) from the Japanese Ministry of Education, Culture, Sports, Science, and Technology (MEXT). This work was also supported by JSPS KAKENHI grant (16K13945 to S.F., JP20H05865 to T.A., JP20K05442 and JP20H04655 to T.T.), JST CREST (JPMJCR18I4 to T.A.), and the 'Dynamic Alliance for Open Innovation Bridging Human, Environment and Materials' from MEXT (T.A.). M.S. gratefully acknowledges a research grant from the Yamada Science Foundation.

\section{Author contributions}

M.K. and K.H. conducted the synthetic experiments and analyzed the data. J.W. performed thermal and electrical measurements. N.H. carried out the single-crystal X-ray diffraction analysis. T.T. performed DFT calculations. J.K. and Y.S. conducted SHG measurement. S.F., M.S. and T.A. prepared the manuscript. S.F. and T.A. conceived the project, which was directed by S.F., M.S. and T.A.

\section{Competing interests}

The authors declare no competing interests.

\section{Additional information}

The online version contains supplementary material available at https://doi.org/10.1038/ s41467-021-21019-4.

Correspondence and requests for materials should be addressed to S.F., M.S. or T.A.

Peer review information Nature Communications thanks Stephen Ducharme and the other, anonymous, reviewer(s) for their contribution to the peer review of this work. Peer reviewer reports are available.

Reprints and permission information is available at http://www.nature.com/reprints

Publisher's note Springer Nature remains neutral with regard to jurisdictional claims in published maps and institutional affiliations.

Open Access This article is licensed under a Creative Commons Attribution 4.0 International License, which permits use, sharing, adaptation, distribution and reproduction in any medium or format, as long as you give appropriate credit to the original author(s) and the source, provide a link to the Creative Commons license, and indicate if changes were made. The images or other third party material in this article are included in the article's Creative Commons license, unless indicated otherwise in a credit line to the material. If material is not included in the article's Creative Commons license and your intended use is not permitted by statutory regulation or exceeds the permitted use, you will need to obtain permission directly from the copyright holder. To view a copy of this license, visit http://creativecommons.org/ licenses/by/4.0/

(C) The Author(s) 2021 